\title{
International partnerships to foster growth in emergency medicine in Romania
}

\author{
Valerie F. Krym, MD, MPH; ${ }^{*}{ }^{\ddagger}$ Rodica Retezar, $\mathrm{MD}_{;}{ }^{\S}$ Steve Scott, $\mathrm{RN}^{\ddagger}$
}

\section{ABSTRACT}

Emergency medicine (EM) is a growing specialty internationally, but has yet to be cultivated in countries with otherwise advanced health care systems. To promote the growth of EM, emergency physicians from countries where EM is a mature specialty can assist with knowledge transfer and development of emergency medical systems to make a lasting impact on the community served. We present our experience in assisting the development of emergency medicine in a university-affiliated regional hospital in Romania.

The International Emergency Medicine Fellowship program at the University of Toronto established links in Cluj-Napoca, Romania, in order to provide expertise and assist local leadership to stimulate growth in EM. In October 2005, a 3-member team travelled to Cluj-Napoca to provide input on how to best use a new physical plant, to deliver continuing education to physicians and nurses, and to help establish long-term development goals. The team identified the following priorities through a comprehensive needs assessment: physical plant organization and patient flow, staffing, staff education, equipment, medication and supplies, and infection control practices. In conjunction with local counterparts, a plan was developed addressing each priority.

Staff, hospital administration and university officials report positive growth in EM as a result of the collaboration. Further work is underway to implement the plan, including international exchange trips to promote mutual growth between partners.

Keywords: international emergency medicine, international collaboration, needs assessment, knowledge transfer, Romania

\section{RÉSUMÉ}

La médecine d'urgence est une spécialité qui prend de l'ampleur dans le monde, mais qu'il faut cultiver dans des pays qui, par ailleurs, ont des systèmes de soins de santé avancés.
Pour favoriser son expansion, les médecins d'urgence de pays où cette spécialité est bien implantée peuvent contribuer au transfert des connaissances et au développement de systèmes de médecine d'urgence en vue d'avoir un impact durable sur la communauté desservie. Nous présentons notre expérience en matière d'aide au développement de la médecine d'urgence dans un hôpital régional de Roumanie affilié à une université.

Le programme de stage postdoctoral en médecine d'urgence internationale de I'Université de Toronto a établi des liens avec la ville de Cluj-Napoca, en Roumanie, afin de lui offrir une expertise et d'aider les intervenants clés locaux à stimuler l'expansion de cette discipline. En octobre 2005, une équipe de trois s'est rendue dans cette ville pour présenter des recommandations sur l'utilisation optimale du nouvel établissement, fournir une formation continue aux médecins et aux infirmières et définir des objectifs de développement à long terme. L'équipe a établi les priorités suivantes dans le cadre d'une évaluation globale des besoins : organisation de l'établissement physique et circulation des patients, dotation en personnel, formation du personnel, matériel, médicaments et fournitures, et mesures de contrôle des infections. Conjointement avec leurs homologues locaux, un plan a été élaboré pour adresser chacune de ces priorités.

Le personnel, I'administration de I'hôpital et des dirigeants de l'université ont constaté que cette collaboration a engendré une croissance positive de la médecine d'urgence. D'autres travaux sont en cours pour la mise en œuvre du plan, y compris des échanges internationaux visant à promouvoir la croissance mutuelle des partenaires.

\section{INTRODUCTION}

Emergency care is growing internationally, but emergency medicine (EM) has not yet been cultivated as a specialty in developing countries or in many countries

From the *Centre for International Health, Faculty of Medicine, University of Toronto, Toronto, Ont., the tDepartment of Family and Community Medicine, Faculty of Medicine, University of Toronto, Toronto, Ont., the $¥$ Department of Emergency Services, Sunnybrook Health Sciences Centre, Toronto, Ont., and the §Department of Medicine, Faculty of Medicine, University of Toronto, Toronto, Ont.

Presented at the 4th Mediterranean Emergency Medicine Congress, Sorrento, Italy, on Sep. 18, 2007, and the First Emergency Medicine in the Developing World Conference in Cape Town, South Africa, on Oct. 4, 2007.

Submitted Jul. 6, 2008; Revised Feb. 6, 2009; Accepted Feb. 10, 2009

This article has not been peer reviewed. 
with advanced health care systems. There are opportunities to help EM grow as a specialty internationally. We present our experience in international EM in a university-affiliated regional hospital in Romania.

\section{Health care in Romania}

Romania is situated in Eastern Europe with a population of approximately 21.7 million. Demographic, socioeconomic and health status indicators are listed in Table 1., ${ }^{1,2}$ In 1998, health care was transformed from a state-financed model to an insurance-based system. ${ }^{3}$ Insurance is mandatory and payroll contributions are the main source of health sector revenue. Public expenditure on health in Romania account for $5.1 \%$ of the gross domestic product, ${ }^{1,3}$ the lowest in Eastern Europe.

From 1994 to 2004, 1.5 million life years were lost because of premature death in Romania. ${ }^{4}$ For men, the leading causes of premature death were, in order of prevalence, accidents, cardiovascular disease and cancer. For women, the leading causes were, in order of prevalence, cancer, cardiovascular disease and accidents. ${ }^{5}$

\section{Emergency care in Romania}

Emergency care in Romania began in 1989. To reduce the fatality rates in trauma and cardiac emergencies, the national government provided ambulances and extrication equipment to regions. Romania's regional governments, the Swiss government and a World Bank loan funded initial training for emergency personnel. ${ }^{6}$

\begin{tabular}{|lc|}
\hline \multicolumn{2}{|l|}{ Table 1. Romania: selected demographic, socioeconomic } \\
and health status indicators \\
\hline Indicators & Value \\
\hline Population (2006) & 532000 \\
Annual growth rate, \% & -0.4 \\
Population aged $\geq 60 \mathrm{yr}, \%$ & 18.9 \\
Life expectancy, yr & \\
$\quad$ Male & 68 \\
$\quad$ Female & 76 \\
Under-5 mortality rate/1000 live births & 16 \\
Infant mortality rate/1000 live births & 16 \\
Maternal mortality rate/100 000 live births & 58 \\
Tuberculosis, prevalence/100 000 population & 128 \\
Physicians, density/1000 population & 1.9 \\
Adult literacy rate, \% & 97.3 \\
GDP/capita, PPP international \$ & 10150 \\
GDP spent on health care, \% & 5.5 \\
Public:private health care expenditure, \% & $66.1: 33.9$ \\
\hline GDP = gross domestic product; PPP = purchasing power parity. & \\
\hline
\end{tabular}

However, many regions did not receive funding and there was no provision for maintenance of skills. Postgraduate (i.e., residency) EM training began in 1993 with the first formal emergency departments (EDs) established in 1997. There is no national nursing curriculum for emergency care.

The lack of central oversight, insufficient equipment and supplies, and the lack of standards and quality assurance training are all obstacles to the delivery of emergency care in Romania. The goals of our project were to perform a needs assessment and assist local leadership in developing EM locally and regionally.

\section{PROJECT SETTING}

Cluj-Napoca is Transylvania's regional capital, located in northern Romania, $480 \mathrm{~km}$ northwest of Bucharest, the nation's capital. With a population of 330000 , ClujNapoca is a major centre for higher learning, culture and industry. The Cluj County Hospital $(\mathrm{CCH})$ is the academic teaching centre affiliated with the "Iuliu Hatieganu" University of Medicine and Pharmacy (UMF) and serves a catchment area of 600000 people. The $\mathrm{CCH}$ has 1592 beds and is composed of many separate stand-alone specialty-specific buildings with inpatient services, referred to as clinics.

The UMF EM residency program was established in 1993. Following medical school, the program lasts 5 years in duration and accepts 12 first-year residents annually. The CCH's ED is the main teaching site for the EM residents. It has an annual census of 16000 adult patients, including approximately 2000 trauma patients per year. Pediatric patients are treated in a separate pediatric hospital.

The ED was designated as the regional trauma and cardiac care centre. There were 8 physicians and 18 nurses assigned to the ED. All physicians had completed advanced cardiac and advanced trauma life support courses. One physician had formal EM training, one in anesthesiology and 6 in primary care. Nurses had completed a formal nursing program, and 5 received on-the-job training in emergency nursing.

\section{International EM initiative}

In 2005, the International Emergency Medicine Fellowship program at the University of Toronto established a partnership with UMF and the CCH's ED. We contacted the ED medical director to identify areas where input and expertise from external consultants 
could stimulate growth and improve EM care. He requested assistance to optimize a new physical plant, provide education to staff and establish long-term development goals.

In October 2005 we took a 3-member team (the fellowship director, a fellow and an emergency nurse) to Cluj-Napoca. We met with stakeholders from the ED, the $\mathrm{CCH}$ and the university to identify priorities. The needs assessment focused on organization of the physical plant and patient flow, staffing, staff education, equipment, medication and supplies, and infection control practices.

\section{Organization of physical plant and patient flow}

The new ED (Fig. 1) opened in September 2005, a few weeks before our arrival. It is located on the first floor of a stand-alone 3-story building, which included a large open area, a resuscitation room with 4 stretchers, and a separate 8 -bed observation area. There were also dedicated rooms for isolation, casting, suturing and ultrasonography. An area was designated for radiography, but was incomplete because of a lack of funds. The top floor housed the laboratory, offices, a classroom and sterilization facilities.

Ambulatory patients arrived on the ground floor, where they were registered by a nurse, and waited in a small waiting room until a stretcher became available. Patients arriving by ambulance were transported directly to the first floor and placed in the next available stretcher and registered, then assessed by a physician.

Patient entry and registration on 2 separate floors hindered communication and patient flow, making

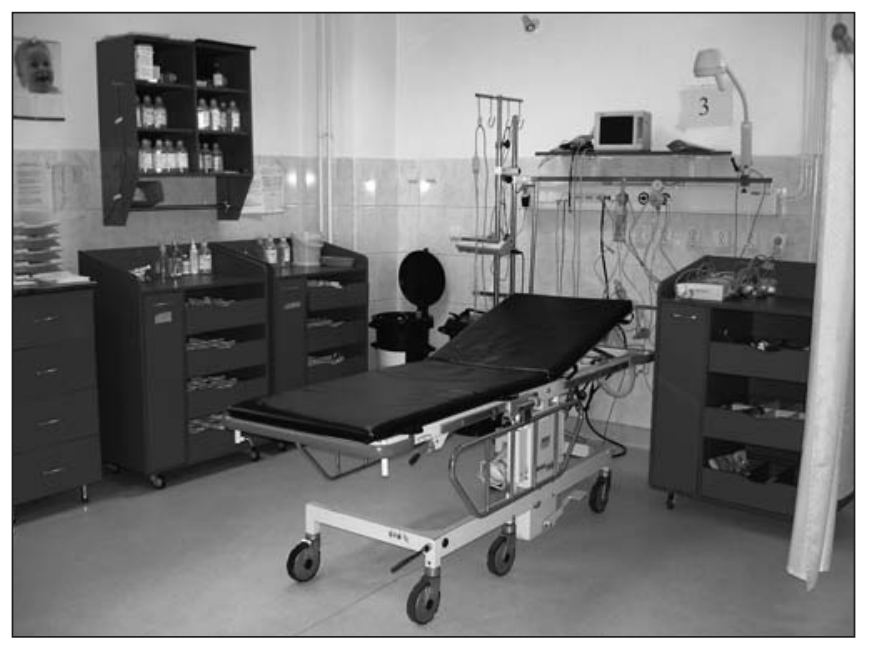

Fig. 1. Resuscitation and monitoring area in the new emergency department of the Cluj County Hospital. effective patient triage impossible. Stretcher allocation depended on the mode of arrival, not patient acuity. Patients with minor problems were often treated in an acute treatment area because of the lack of a minor treatment area. This often delayed care for the severely ill. The stand-alone building posed challenges for staff because patients had to be transferred from one clinic building to another to have imaging (plain radiography, computed tomography), see a consultant, be admitted to a ward or undergo surgery.

We proposed improvements to the layout and the need for a triage system. As a first step, we suggested reorganizing treatment areas so all patients would enter and be assessed on one level. Staff agreed that ambulatory patients could be accommodated on the first floor and agreed on the need for patient triage. Because of the scope of the proposed reorganization and implementation of a triage system, these items were deferred to a future project.

The medical director identified a lack of data about patient volume and case-mix. He also sought suggestions on charting, data collection and basic data interpretation for audit purposes. A clerk recorded the discharge diagnosis and disposition of each patient in a central database. We assisted the medical director to review this data and proposed capture of additional information to meet his needs. The ED record was a 1-page form with no space for nursing notes. As a first step, we proposed revisions to the ED record, including separate areas for nurses and physicians notes. We provided examples of other ED records so local leadership could develop new charting tools to suit their needs, and we helped them complete an initial draft of a new ED record.

\section{Staffing}

The medical director asked us for staffing recommendations. Based on patient volume and acuity, we determined that there were sufficient physicians, residents, porters and cleaning staff; however, more nurses were required. We discussed ways to address this need and provided options to increase the number and placement of nurses. Emergency department leadership developed a recruitment and retention plan that targeted experienced nurses elsewhere in the hospital, highlighting on-the-job training offered in the ED. We proposed expanding the nursing scope of practice, including nurse-based triage, and reallocating patient registration to a clerk. 
Job descriptions for nurses were available, but required updating to incorporate the new skills that were proposed as part of the expanded scope of nursing practice. We also assisted with updates of job descriptions for physicians and other ED staff. We also recommended name tags for all personnel to improve the public's ability to identify staff in their respective roles.

\section{Staff education}

Before our arrival, staff lacked continuing medical education opportunities. To address this need, we prepared 26 didactic and hands-on teaching sessions. Topics included key EM concepts, such as advanced airway management, acute coronary syndrome, new advanced cardiac life support protocols, management of the trauma patient, medical documentation and clinical quality improvement. All sessions were delivered with simultaneous translation by a team member fluent in Romanian (Fig. 2). We also provided staff with lecture handouts, written in Romanian.

Many tasks carried out by nurses in industrialized countries were still performed by physicians. To the nursing staff, we described the role of the ED nurse and delivered didactic and practical sessions on patient history-taking, physical assessment of patients and nursing pharmacology. We provided hands-on training in basic and advanced cardiac life support, invasive monitoring, and ECG interpretation. Nurses quickly identified the opportunity to expand their current role, and began working with physicians to identify tasks that nurses could assume.

The EM residents obtain most of their knowledge and training "on the job" despite the presence of a formal training program. The residents identified the need for didactic teaching sessions and case presentations

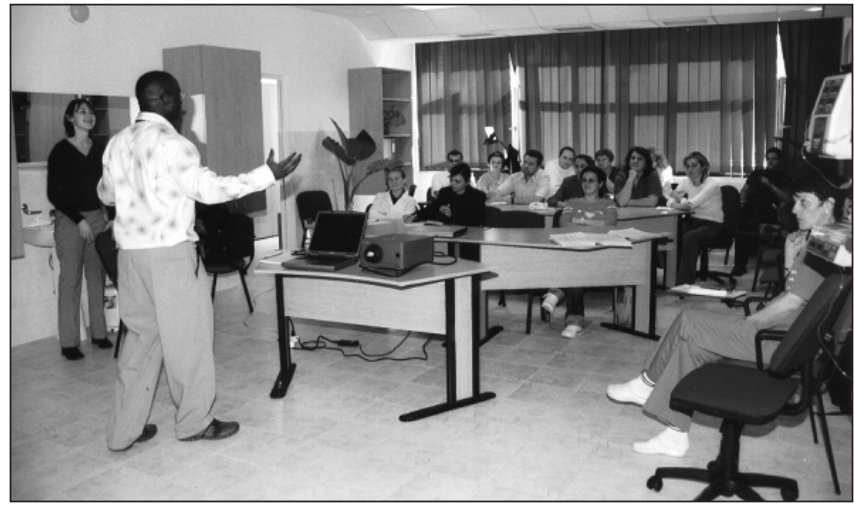

Fig. 2. Didactic teaching session for nursing staff, with simultaneous translation. similar to an "academic half-day" in Canadian EM residency programs. We introduced these concepts to the residents and staff physicians by presenting rounds on specific topics requested by the physicians and residents. Local ED leadership is now implementing an ongoing continuing medical education program for all physicians and trainees.

\section{Equipment}

The CCH had funding for a new physical plant but lacked funds for medical equipment and other furnishings. For example, there were 2 old cardiac monitors, but 4 additional monitors and a defibrillator were required for the acute care area. Essentials, such as stethoscopes and sphygmomanometers were in short supply. The ED also required items such as an otoscope ophthalmoscope and blood glucometer.

We helped develop a list of recommended equipment and identified potential sources of funding. Subsequent to our departure, the $\mathrm{CCH}$ obtained large capital grants from the Romanian government and the World Bank to purchase cardiac monitors, ventilators, stretchers and other equipment for the ED.

\section{Medication and supplies}

The ED lacks sustained financial resources to meet their medication and medical supply needs. Most essential medications are available, but often in limited quantities. We helped administration prioritize immediate and ongoing essential medication and supply requirements based on the World Health Organization list of essential drugs ${ }^{7}$ deemed necessary in the ED. Priority items were then sourced and purchased at the best possible price. The list would also be used to target potential donors to help fund the department.

Consumable supplies were allocated to the ED by a central supply department, but did not meet daily requirements. For example, only a limited number of intravenous tubing sets were given to be used each day, and the ED often ran out. We helped the medical director address this problem by determining requirements based on volume and case-mix, and by bringing $\mathrm{ED}$ and hospital administration together to develop a long-term solution.

\section{Infection control practices}

Housekeeping staff worked hard to keep the ED clean. 
Despite the cleanliness, we noted there was a chronic shortage of procedure gloves, surgical gloves and masks. This presented risks to staff and other patients. We taught infection control practices and personal protection against communicable disease in our lecture series. We also acquired a local supply of gloves and masks, but acknowledged this was a temporary solution that could not be sustained because of a lack of funds.

Health care staff washed their hands frequently. Disposable paper towels were not available and there was only 1 hand towel available to dry their hands. We recommended the hand towel be changed frequently and regularly. Although this was viewed as a compromise, it was an improvement over current practice.

\section{DISCUSSION}

There is a tendency for visiting medical consultants to conduct needs assessments and develop plans without consulting local stakeholders. When performing international work, the most useful skill is the willingness to listen to the needs of local staff. ${ }^{8}$ Developing trust and a solid working relationship with local counterparts are essential first steps. Only then can consultants make informed decisions, provide culturally appropriate recommendations and build important relationships within the local medical community. Finally, teaching local staff ensures sustainability when staff retains and uses the new knowledge and teaches others.

A local champion should be identified early. Ideally, the local champion should have the authority, motivation and willingness to help lead the project and be the key contact. We identified the ED medical director as the local champion because he was willing to participate, he was authorized to make change, and was well respected by hospital administration and regional government. Meetings should take place with all key stakeholders and decision-makers, such as hospital administrators, university officials and government leaders. It is also important to hold discussions with health care staff who work on the "front lines," as well as the patients they serve.

The ability to communicate in the local language can overcome many of the barriers that occur in international work. The International Emergency Medicine Fellow who was part of our project spoke Romanian. Because we provided our Romanian hosts with translation of lectures and teaching materials, interactions with our team were encouraged. Having a team member familiar with local customs also gave us a better understanding of their nonverbal communication and helped minimize any misunderstandings that might occur in a cross-cultural setting.

Cultural diplomacy and sensitivity are essential in the international setting. Observation often yields information on how and why a system works. We discovered that local counterparts appreciated immediate feedback, recommendations and initial results. A formal presentation at the project's conclusion, incorporating photos of local staff making positive changes, was well received. It gave local staff a sense of pride and ownership.

We identified several steps that are needed before implementing a new initiative. First, it must address a real need, ask what local counterparts see as important and what is achievable, and work with them to define their priorities. Recommendations must be culturally appropriate, sensitive to local needs and sustainable in that setting. They should not be transplanted from elsewhere. To ensure long-term success, the local champion and staff must be empowered and have a sense of ownership of the initiative. Finally, if it requires funding, the funding source must be secured. Complicated, expensive initiatives, those not meeting a local need, or those without local buy-in are not likely to succeed. The goal of an international project should be partnership and collaboration leading to meaningful, sustainable change.

\section{CONCLUSION}

This international EM project promoted knowledge transfer in emergency care in northern Romania. Direct feedback and evaluations from staff indicated they viewed the 1-month project positively, and believed it facilitated positive, sustainable change in EM. More importantly, this initial project established trust, and established the basis for a long-term relationship that could foster further growth. Future plans include a second project to help implement a triage system adapted for local conditions and staff exchange trips to promote exchange of knowledge between partners.

Acknowledgements: The authors wish to thank Dr. David Zakus, the Centre for International Health at the University of Toronto and the Academic Emergency Physicians Group at the Sunnybrook Health Sciences Centre for their support of this International EM project. The authors also thank the Academic Emergency Physicians Group from Mount Sinai Hospital for their support of the International EM Fellowship program. The authors also wish to thank Dr. Dorin Muresan, the medical director, and the staff at Cluj Country ED for their hospitality, collaboration and support during this project. 
Competing interests: None declared.

Funding: Dr. Krym is supported by the Centre for International Health, University of Toronto, and the Academic Emergency Physicians Group, Sunnybrook Health Sciences Centre, Toronto, Ont.

\section{REFERENCES}

1. Romania. Geneva (CH): World Health Organization; 2009. Available: www.who.int/countries/rou/en (accessed 2009 Sep 25).

2. Romania: country profile. Bucharest (RO):United Nations Development Programme Romania; 2003. Available: www .undp.ro/profile_romania.php (accessed 2009 Sep 25).

3. Bara A-C, van den Heuvel W, Maarse JAM. Reforms of the health care system in Romania. Croat Med J 2002;43:446-52.

4. Human health and the environment. In: Environmental performance review for Romania: buman bealth and the environment. Geneva (CH): United Nations Economic Commission for Europe; 2000. Available: www.unece.org/env/epr /epr_studies/romania.pdf (accessed 2009 Oct 1).

5. Potential years of life lost (Pyll) due to cancer in Romania in 2004. Washington (DC): UICC World Cancer Congress; 2006. Available: http://2006.confex.com/uicc/uicc/techprogram /P2710.HTM (accessed 2009 Sep 25).

6. Romania - a bealth sector reform project, implementation completion report. Washington (DC): World Bank; 2004. Available: www-wds.worldbank.org/external/default/WDSContentServer /WDSP/IB/2005/01/03/000090341_20050103114253/Rendered /PDF/30301.pdf (accessed 2009 Oct 1).

7. Essential medicines: WHO model list. Geneva (CH): World Health Organization; 2005. Available: http://whqlibdoc .who.int/hq/2005/a87017_eng.pdf (accessed 2009 Sep 25).

8. Mullan F, Panosian C, Cuff P, editors. Healers abroad: Americans responding to the buman resource crisis in HIV/AIDS. Washington (DC): National Academies Press; 2005.

Correspondence to: Dr. Valerie Krym, Department of Emergency Services, Sunnybrook Health Sciences Centre, 2075 Bayview Ave., Toronto ON M4N 3M5; v.krym@utoronto.ca

\section{LOO KING FOR A NEW JOB?}

In print and online, the best way to advertise or find employment opportunities in academic, administrative or community emergency medicine is through the Canadian Journal of Emergency Medicine.

Print version of CJEM: The deadline for advertising in the print version of CJEM is one month before issue date. For ad rates and further information, please contact Beverley Kirkpatrick or Deborah Rodd at CMA Publications (800 663-7336 or 613 731-8610 x2127/2314, advertising@cma.ca).

\section{Looking for an Emerg Doc?}

SPECIAL BONUS: As a bonus to print advertisers, $C J E M$ is pleased to offer website advertising space on CAEP's website (www.caep.ca) free of charge.

CAEP website alone: Advertising in the CAEP website alone is billed at a rate of $\$ 100$ a month, and can be obtained at any time. Please contact CAEP's Head Office for advertising solely on the website (800463-1158 or advertising@caep.ca). 